\title{
Subtle Imaging Findings Aid the Diagnosis of Adolescent Hereditary Spastic Paraplegia and Ataxia
}

\author{
Franca Wagner ${ }^{1} \cdot$ David S. Titelbaum ${ }^{2} \cdot$ Renate Engisch ${ }^{3} \cdot$ Emily K. Coskun $^{4} \cdot$ Jeff L. Waugh ${ }^{5}$ \\ Received: 22 October 2017 / Accepted: 11 January 2018 \\ ๑) Springer-Verlag GmbH Germany, part of Springer Nature 2018
}

\begin{abstract}
Purpose Hereditary spastic paraplegia (HSP) and hereditary spastic ataxia (HSA) are a heterogeneous group of genetic disorders characterized by progressive lower limb spasticity resulting from pyramidal tract dysfunction. By identifying critical imaging findings within the clinical context of spasticity, radiologists are uniquely positioned to recommend specific genetic testing, and thus facilitate diagnosis.

Methods We present two examples of HSP and HSA that had gone clinically unrecognized for years, and in which magnetic resonance imaging played a critical role in the diagnosis.

Results Radiologists' awareness of HSP and HSA, combined with a critical review of the clinical history and characteristic imaging findings led to specific genetic testing and a definitive diagnosis.

Conclusion Awareness of HSP and HSA among radiologists will expedite more accurate diagnosis, explanation of patient symptoms, recommendation for syndrome-specific treatment, and family planning considerations.
\end{abstract}

Keywords ARSACS $\cdot$ SPG11 $\cdot$ HSP $\cdot$ HSA

\begin{tabular}{ll}
\multicolumn{2}{l}{ Abbreviations } \\
ALS & Amyotrophic lateral sclerosis \\
ARSACS & $\begin{array}{l}\text { Autosomal recessive spastic ataxia of Charle- } \\
\text { voix-Saguenay }\end{array}$ \\
& Corticospinal tract \\
CST & Diffusion tensor imaging \\
DTI & Fractional anisotropy \\
FA & Hereditary spastic ataxia \\
HSA & Hereditary spastic paraplegia \\
HSP & Middle cerebellar peduncle \\
MCP & Spastic paraplegia \\
SPG & Thin corpus callosum \\
TCC &
\end{tabular}

$\triangle$ Franca Wagner

franca.wagner@insel.ch

1 Department of Diagnostic and Interventional Neuroradiology, University Hospital of Bern, University of Bern, Bern, Switzerland

2 Shields Healthcare, Brockton, MA, USA

3 Department of Radiology, Luzerner Kantonsspital, Lucerne, Switzerland

4 Harvard Vanguard Medical Associates, Boston, MA, USA

5 Department of Neurology, Massachusetts General Hospital, Boston, MA, USA

\author{
TPF Transverse pontine fibers \\ WMH White matter hyperintensity
}

\section{Introduction}

Hereditary spastic paraplegia (HSP) and hereditary spastic ataxia (HSA) are a heterogeneous group of genetic disorders characterized by progressive lower limb spasticity resulting from pyramidal tract dysfunction. These disorders may be associated with other neurological signs including ataxia, dysarthria, peripheral neuropathy, seizures and intellectual disability [1-3]. While no curative treatments are currently available, accurate diagnosis provides patients and families an explanation for progressive neurological impairment, guides prognosis, and informs about family planning. Diagnosis is often difficult due to slowly progressive clinical findings and imaging findings that can go unrecognized. By being able to identify critical imaging findings within the clinical context of spasticity, radiologists are uniquely positioned to recommend specific genetic testing, and thus facilitate diagnosis. We present two examples of patients with HSP and HSA that had gone clinically unrecognized, and in which radiology played a critical role in the diagnosis. It is hoped that a greater awareness of HSPs and HSAs 
among radiologists will result in more accurate diagnosis in the future.

\section{Methods}

A case review of magnetic resonance imaging (MRI) examinations of two patients referred to the neurology department to evaluate spasticity was conducted. Imaging was performed on a 1.5T General Electric (Milwaukee, WI, USA) HDxT scanner. Diffusion tensor imaging (DTI) was performed on both patients and analyzed with FUNCTOOL software, with generation of directional and fractional anisotropy (FA) maps and fiber tractography. Based on the findings of these examinations, specific genetic testing was recommended.
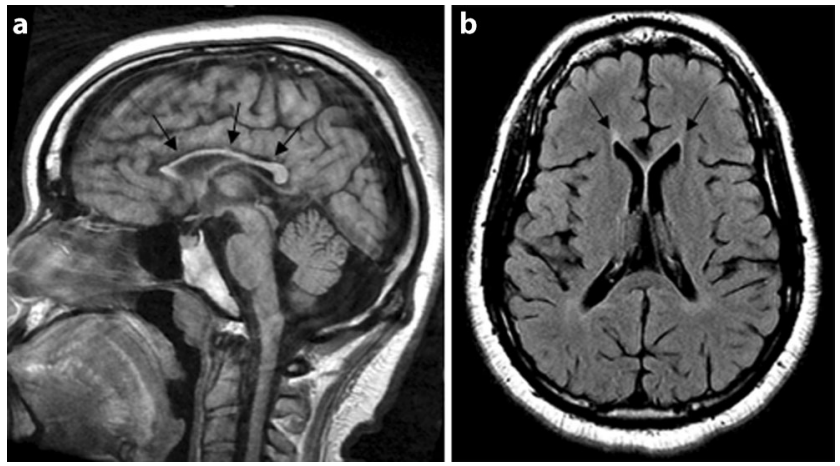

Fig. 1 A 21-year-old male with spasticity and gait disturbance found to have SPG11. Sagittal T1-weighted image (a) exhibits marked thinning of the corpus callosum (arrows). Axial FLAIR image (b) shows periventricular white matter hyperintensities extending anteriorly from the frontal horns, referred to as the "ears of the lynx" sign (arrows)
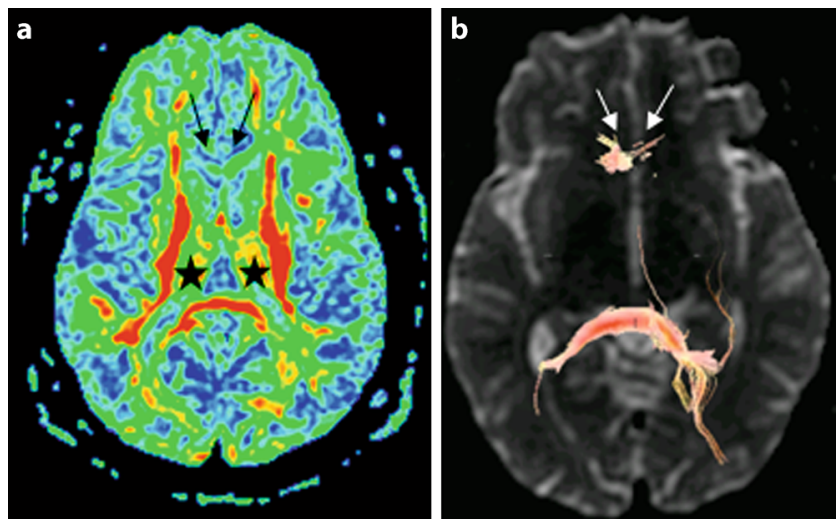

Fig. 2 Diffusion tensor imaging of the same patient with SPG11 as in Fig. 1. Fractional anisotropy (FA) map (a) reveals severely reduced FA of the genu of corpus callosum (arrows) and marked attenuation of the splenium as well as the cortical spinal tracts (asterisks). Selective fiber tractography (b) also shows severe reduction of anterior callosal tracts (arrows)

\section{CASE 1: Spastic Paraplegia Gene 11 (SPG11)}

A 21-year-old American male presented to the neurology clinic with a 2-3 year history of progressive gait imbalance and bilateral leg weakness. He had sat, crawled, and walked at appropriate ages, but had a history of cognitive developmental delay and did not speak until age 4-5 years. He required leg bracing for bowlegs at the age of 2 years and was seen in a cerebral palsy clinic for tight hamstrings, when he was 10 years old. He was seen in a pediatric neurology clinic and laboratory tests for urine organic acids and FMRl gene sequencing were normal. The cause of his cognitive developmental delay remained unknown. Neuropsychiatric testing at age 15 years showed mild to moderate mental retardation.

He had normal balance without any unusual number of falls until the age of 18 years. At that time, he sustained a left ankle injury while playing football, and subsequently developed knee instability and progressive gait abnormality. He was initially seen in a sports medicine clinic where he was diagnosed with Achilles tendonitis and patellofemoral syndrome. He continued having frequent falls, and developed bilateral leg weakness, leg cramps, and paresthesia of both feet. On neurological examination at age 21 years, he was found to have dysarthria and slowed but fluent speech. He had spasticity in both legs and significant weakness of the bilateral iliopsoas muscles and hamstrings. There was reduced pinprick sensation in his hands and distal feet. He had a wide-based, very unsteady gait and appeared to throw his legs out when walking.

Brain MRI revealed a markedly thin corpus callosum (TCC), most pronounced at the genu and body, best seen on sagittal T1-weighted (T1W) sequences (Fig. 1a). Axial T2-fluid-attenuated inversion recovery (FLAIR) images revealed subtle linear white matter hyperintensities (WMHs) extending anteriorly from the frontal horns of the lateral ventricles (Fig. 1b) without restricted diffusion. The DTI showed severe attenuation of the corpus callosum and cortical spinal tracts (CSTs) (Fig. 2a, b). Cervical and thoracic spine MRIs were unremarkable, showing no cord lesions or atrophy. The electromyography/nerve conduction study showed electrophysiological evidence for a predominantly axonal mild sensory polyneuropathy. There was no evidence of myopathy. Human T-lymphotropic virus types I and II (HTLV I/II) antibody testing were negative. Based on the brain abnormality detected by MRI, genetic testing for hereditary spastic paraplegia was conducted. This showed two autosomal recessive heterozygous frameshift mutations in the SPG11 gene (c.3320_3321: 2 bp deletion of GT and c.4307_4308: 2 bp deletion of AA).

After the final diagnosis the patient was prescribed baclofen up to $10 \mathrm{mg}$ three times a day, resulting in poor muscular tone, and subsequently adjusted to $5 \mathrm{mg}$ three times 

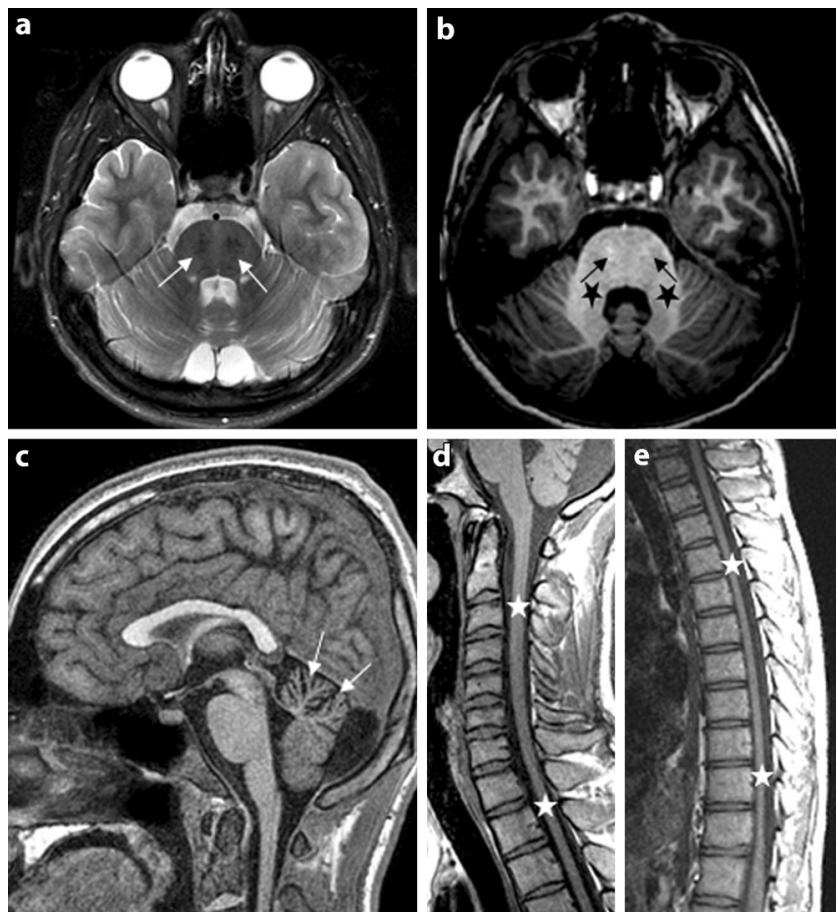

Fig. 3 A 17-year-old male with progressive lower leg rigidity and bradykinesia found to have ARSACS. Axial T2-weighted image (a) shows horizontal pontine striations in the region of the cortical spinal tracts (arrows). Axial-T1 weighted image (b) shows pontine and middle cerebellar peduncle hypertrophy (asterisks). Small areas of increased T1 signal are seen in the cortical spinal tracts (arrows) suggesting hypermyelination. Sagittal T1-weighted images of the brain (c) exhibit superior vermian atrophy (arrows). Cervical (d) and thoracic (e) spine MRI (sagittal T1) showed pronounced diffuse cord atrophy (asterisks)

a day. He was referred to physical therapy and is being fitted for bilateral custom ankle foot orthosis, and has a rolling walker.

\section{CASE 2: Autosomal Recessive Spastic Ataxia of Charlevois-Saguenay (ARSACS)}

A 15-year-old male student of mixed European heritage presented with a complaint of slow movements and stiffness of the legs. At 2 years of age, he had been diagnosed with cerebral palsy due to delayed ambulation, leg stiffness, and frequent falls; however, his prenatal and perinatal history, and subsequent MRIs of the brain (at ages 2, 8, 12 , and 13 years) were reported to be normal. His mother was told cerebral palsy was a "catch all" diagnosis. Worsening of his lower extremity stiffness led to an MRI of the lumbar spine when he was 2 years old. The diagnosis was a thin fatty filum with the conus at the L1-2 level. Surgical release led to rapid improvement in his ease and speed of ambulation. Notably, this surgery did not entirely normalize his lower extremity muscle tone and left his elevated up- per extremity tone unchanged. Between the ages of 3 and 15 years, the elevated muscle tone progressed very slowly, producing only mild limitations related to slow ambulation, a mild increase in fatigue when writing, and a longer time to work through his muscle stiffness in the mornings or after prolonged sitting. He actively participated in school sports, although his slowed movements led him to prefer solo sports (golf) to team sports. On his initial visit to the movement disorders clinic, his extremity tone (legs $>$ arms) was notable for rigidity without spasticity, mild bradykinesia of gross and fine movements. His strength, sensation and reflexes were normal, as were his intellect, attention and memory. His language and speech were normal, but speech production was delayed and slightly slowed, in keeping with the pace of his other movements. Based on his rigidity and bradykinesia, he was diagnosed with Parkinsonism. Trials of levodopa and ropinirole showed no benefit and the treatments were discontinued due to gastrointestinal and mood side effects, respectively. Subsequently, a trial of baclofen led to substantial improvement in the ease and speed of movement.

The patient was followed in our movement disorder clinic for 2 years during which there was no clear progression in symptoms. Tone and speed of movement were always moderately abnormal, but reflexes were consistently normal. On routine follow-up at 2.5 years following presentation, he was found to have pathologically brisk reflexes at the knee and ankle, and bilateral upgoing toes in response to Babinski testing. This finding prompted a repeat MRI of the brain and spinal cord. Axial T2 (Fig. 3a) and $\mathrm{T} 1$ images (Fig. 3b) revealed pontine enlargement with non-enhancing horizontal linear T2 hypointense and T1 hyperintense striations without restricted diffusion, suggesting hypermyelination of the transverse pontine fibers (TFPs). Other findings included middle cerebellar peduncle (MCP) hypertrophy, superior vermian atrophy and thinning of the posterior body of corpus callosum (Fig. 3c). Cervical and thoracic spine MRI showed pronounced cord atrophy (Fig. 3d, e). No recurrence of tethered cord was seen on lumbar spine MRI. The DTI revealed hypertrophied TFPs that appeared to constrict and splay the CSTs (Fig. 4). In retrospect, pontine striations could be traced at least as far back as age 9 years, but had not been recognized during the earlier studies.

The suspicion of ARSACS based on these imaging abnormalities led to subsequent Sanger sequencing of the SACS gene, which revealed three compound heterozygous mutations. One of these mutations led to replacement of a highly-conserved amino acid and was previously reported in only one ARSACS patient: c.4744G > A (p.Asp1582Asn) [4]. One mutation led to frameshift and premature truncation, disrupting the last 2178 residues: c.7205_7206delTT (p.Leu2402Argfs*6). The final mutation was designated 

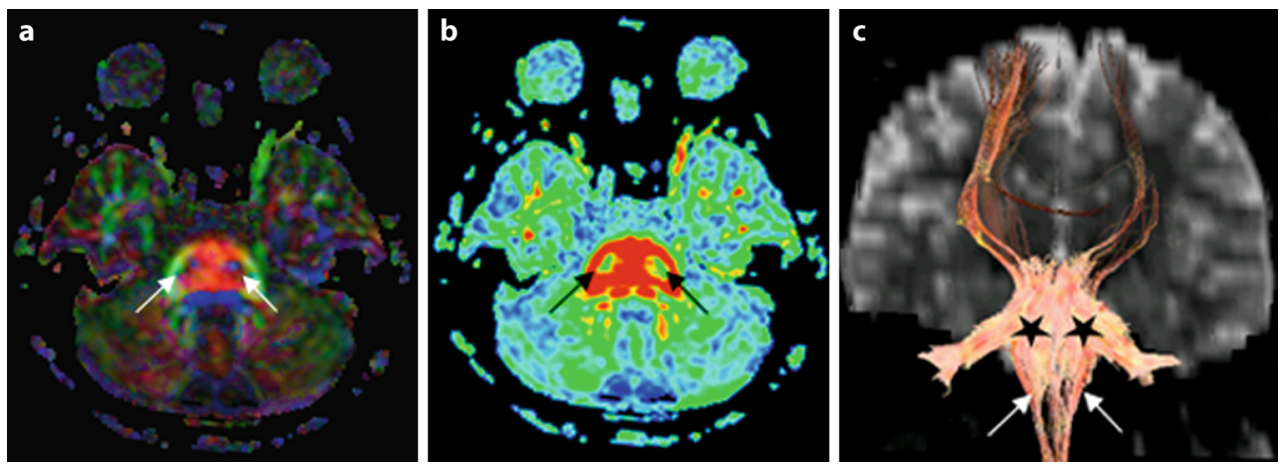

Fig. 4 Diffusion tensor imaging of the same patient with ARSACS as in Fig. 3. Directionally encoded color fractional anisotropy images (a) and FA map (b) reveal small cortical spinal tracts (arrows) compressed by hypertrophic transverse pontine fibers TPFs (shown in red). Tractography (c) reveals markedly increased fiber density within the pons (asterisks), and subsequent decrease of cortical spinal tract density in the cervicomedullary junction (arrows)

a variant of unknown significance, due to its presence in $0.2 \%$ of the population and predictions of pathogenicity varying from likely benign (C0, Align-GVGD) to deleterious (SIFT): c.7528 G>A (p.Ala2510Thr) [4].

After final diagnosis, oral baclofen was started and gradually increased to $10 \mathrm{mg}$ twice per day, providing substantial relief from his spasticity, and he thus far remains stable. The patient has declined intragastrocnemius botulinum toxin injection. Given his late onset and slow progression, a longer period of independent ambulation and a normal lifespan is predicted.

\section{Discussion}

\section{Hereditary Spastic Paraplegia}

The HSPs are a group of genetic syndromes that share common symptoms of slowly progressive leg weakness and spasticity owing to corticospinal tract axonal degeneration. Some HSPs may be complicated by other neurological findings such as ataxia, seizures, developmental delay and bladder dysfunction [1]. The HSP nomenclature follows the convention of "SPG", for spastic paraplegia followed by a number corresponding to the sequential number of gene discovery (e.g.,SPG11). As of 2014, 55 SPG genes had been identified [5]. Mode of inheritance and age of onset of symptoms varies depending on the specific mutation. Presentations are highly variable, even within specific families.

Pathological reports are sparse but have revealed marked symmetric distal CST axonal loss and proximal cord sensory axonal loss, corresponding to length-dependent dyingback axonal pathology [6]. Anterior horn cell loss may also be found [7]. Myelin pallor may be observed, but without the myelin breakdown products typically seen with demyelination [6]. Also noteworthy is that axonal loss occurs without pronounced cord atrophy, limiting the possible role of MRI volumetric cord analysis for determining disease extent and progression [6]. Intracellular pathophysiological mechanisms may include impaired axonal transport, mitochondrial dysfunction and oxidative stress, and abnormalities of lipid and myelination, ultimately resulting in CST axonal degeneration [5]. An autopsy report on a patient with autosomal recessive HSP (ARHSP) with TCC revealed cortical and deep nuclear neuronal loss and gliosis, and white matter gliosis including severe callosal gliosis suggestive of degeneration, as opposed to hypoplasia [7].

The SPG11 is the most common type of ARHSP. It is classified as an ARHSP with TCC and is caused by mutations in spatacsin (also known as KIAA1840, ALS5, or $C M T 2 X)$ a protein of uncertain function. The SPG11 shares the same mutation locus as juvenile amyotrophic lateral sclerosis (ASL) [2, 5, 8-11]. Imaging findings in patients with SPG11 include TCC and WMH extending anteriorly from the frontal horns of the lateral ventricles in a linear or tufted orientation, described as an "ears of the lynx" appearance. The TCC may be misconstrued as callosal hypoplasia or dysgenesis; however, dysgenesis results in hypoplasia of the body and splenium, whereas TCC is characterized by a morphologically intact, albeit severely atrophied corpus callosum [2, 12]. Ears of the lynx WMHs may be elusive as in case 1 , however may be easily dismissed as nonspecific or gliotic WMHs (which generally lack the anterior linear appearance) [13] or may be misdiagnosed as leukodystrophy [14]. New MR spectroscopy studies have shown decreased brain $\mathrm{N}$-acetylaspartate consistent with axonal loss [15] and DTI studies of cerebral white matter have shown increased radial and mean diffusivity, but intact axial diffusivity, suggesting myelin loss. These studies have also shown decreased frontal white matter FA, implying frontal disconnection [16], potentially related to the ears of the lynx WMHs. 


\section{Hereditary Spastic Ataxia}

The HSAs are a heterogeneous group of genetic disorders that result in progressive lower extremity spasticity, ataxia, and may also be associated with dysarthria and extraocular muscle dysfunction. Nomenclature categorizes HSAs as SPAX (spastic ataxia) with a sequential number corresponding to the order of gene discovery. The ARSACS is classified as SPAX6, with mutations in sacsin (gene: SACS), a protein of uncertain function [17]. Initially described in Quebec, other clusters have been described in Eastern Europe, Mediterranean countries, and Japan [17-19]. Other clinical findings seen in patients with ARSACS include lower limb peripheral neuropathy and fundoscopy may reveal hypermyelinated fibers radiating from the optic nerve $[12,20]$. As further cases of ARSACS have been identified and the spectrum of causative mutations has expanded, the range of phenotypes has also broadened, including among Quebecois [4]. Imaging findings in patients with ARSACS often include horizontal pontine tigroid striations, pontine and MCP enlargement, and cerebellar and spinal cord atrophy [12, 21-25]. Cervical cord atrophy appears to be common in the Quebecois population, but uncommon in Belgian patients $[26,27]$. Other findings may include lateral pontine and MCP FLAIR hypointensities/T2 hyperintensities, parietal atrophy, circumscribed thinning of the posterior midbody of the corpus callosum, posterior fossa arachnoid cysts, and unilateral cerebellar hemispheric atrophy [28-30]. The DTI analysis has shown that transverse pontine striations and pontine and MCP enlargement appear to be caused by abnormally enlarged transverse pontocerebellar fibers [29, 31, 32]. On tractography, these hyperplastic fibers appear to compress and interrupt the pyramidal tracts at the pontine level [31]. Striations may be overlooked on MRI, as occurred several times in case 2, and cerebellar and cord atrophy may be considered nonspecific [22-25]. Optical coherence tomography reveals retinal fiber layer thickening [31].

\section{Considerations for HSPs and HSAs}

For both HSPs and HSAs, diagnosis involves exclusion of other causes of paresis, spasticity, and ataxia. These include cerebral palsy, multiple sclerosis, vitamin B12 and copper deficiencies, spinocerebellar ataxia syndromes, amyotrophic lateral sclerosis, leukodystrophies, mitochondrial disorders, HTLV-1-associated myelopathy, infectious and inflammatory encephalopathies, and spinal cord tethering or compression [5, 12]. Imaging thus plays an important role both in excluding alternative causes, and in identifying specific findings, often obscure or difficult to recognize, which may lead to inclusion of HSP or HSA in the differential diagnosis. Radiologists need to be aware that a thinned corpus callosum is different from callosal agenesis, in that it is morphologically intact but small. While callosal degeneration can occur in other diseases such as multiple sclerosis, in the setting of adolescent spasticity a thin corpus callosum is highly suggestive of SPG 11. Likewise, the observation of pontine hypertrophy with horizontal T2-hypointense striations is highly suggestive of ARSACS. The added value of brain MRI in identifying subtle findings in hereditary spastic paraplegias and hereditary spastic ataxias has been also discussed by Goller at al. [33]. The authors indicate that MRI examinations in younger affected patients are usually unremarkable, whereas older patients may demonstrate cortical and perisylvian volume loss and brain stem atrophy. Additionally, we suggest that supplementing conventional MRI with DTI can be helpful in identifying subtle tract pathology, such as in ARSACS where DTI allows visualization of the corticospinal tracts constricted by hypertrophic pontine fibers, and in SPG11 where DTI may demonstrate decreased density of callosal connections.

No definitive treatments are currently available, and treatment is therefore focused on relieving specific symptoms of spasticity, dystonia, and bladder hypertonicity. While HSPs and HSAs are relatively rare, ultimately, CST degeneration may be a final common pathway shared with many other neurodegenerative diseases, including spinal muscular atrophy and ALS. Therefore, expanding the published literature on diagnosed patients may contribute future insights into shared disease processes and treatments, such as targeting specific intracellular pathophysiological mechanisms and gene therapy [5].

\section{Conclusion}

Imaging findings in patients with HSPs and HSAs may be elusive and can easily go undiagnosed or be attributed to other causes. Only an awareness of HSPs and HSAs and a critical review of clinical history and imaging findings can place the radiologist in a position to suggest specific genetic testing. Such radiographically informed testing may lead to definitive, previously unrecognized diagnoses, and thus provide an explanation of patient symptoms, suggest potential prognosis-specific symptomatic treatments, and consideration of family planning needs.

\section{Compliance with ethical guidelines}

Conflict of interest F. Wagner, D.S. Titelbaum, R. Engisch, E.K. Coskun and J.L. Waugh declare that they have no competing interests.

Ethical standards All procedures performed in studies involving human participants were in accordance with the ethical standards of the institutional and/or national research committee and with the 1964 Helsinki declaration and its later amendments or comparable ethical standards. Written informed consent was obtained from both patients. 


\section{References}

1. Harding AE. Classification of the hereditary ataxias and paraplegias. Lancet. 1983;1:1151-5.

2. Paisan-Ruiz C, Dogu O, Yilmaz A, Houlden H, Singleton A. SPG11 mutations are common in familial cases of complicated hereditary spastic paraplegia. Neurology. 2008;70:1384-9.

3. Fink JK. Hereditary spastic paraplegia. Curr Neurol Neurosci Rep. 2006;6:65-76.

4. Thiffault I, Dicaire MJ, Tetreault M, Huang KN, Demers-Lamarche J, Bernard G, Duquette A, Larivière R, Gehring K, Montpetit A, McPherson PS, Richter A, Montermini L, Mercier J, Mitchell GA, Dupré N, Prévost C, Bouchard JP, Mathieu J, Brais B. Diversity of ARSACS mutations in French-Canadians. Can J Neurol Sci. 2013;40:61-6.

5. Lo Giudice T, Lombardi F, Santorelli FM, Kawarai T, Orlacchio A. Hereditary spasticity paraplegia: clinical-genetic characteristics and evolving molecular mechanisms. Exp Neurol. 2014;261:518-39.

6. DeLuca GC, Ebers GC, Esiri MM. The extent of axonal loss in the long tracts in hereditary spastic paraplegia. Neuropathol Appl Neurobiol. 2004;30:576-84.

7. Kuru S, Sakai M, Konagaya M, Yoshida M, Hashizume Y. Autopsy case of hereditary spastic paraplegia with thin corpus callosum showing severe gliosis in the cerebral white matter. Neuropathology. 2005;25:346-52.

8. Shibasaki Y, Tanaka H, Iwabuchi K, Kawasaki S, Kondo H, Uekawa K, Ueda M, Kamiya T, Katayama Y, Nakamura A, Takashima H, Nakagawa M, Masuda M, Utsumi H, Nakamuro T, Tada K, Kurohara K, Inoue K, Koike F, Sakai T, Tsuji S, Kobayashi H. Linkage of autosomal recessive hereditary spastic paraplegia with mental impairment and thin corpus callosum to chromosome 15A13-15. Ann Neurol. 2000;48:108-12.

9. Casali C, Valente EM, Bertini E, Montagna G, Criscuolo C, De Michele G, Villanova M, Damiano M, Pierallini A, Brancati F, Scarano V, Tessa A, Cricchi F, Grieco GS, Muglia M, Carella M, Martini B, Rossi A, Amabile GA, Nappi G, Filla A, Dallapiccola B, Santorelli FM. Clinical and genetic studies in hereditary spastic paraplegia with thin corpus callosum. Neurology. 2004;62:262-8.

10. Stevanin G, Montagna G, Azzedine H, Valente EM, Durr A, Scarano V, Bouslam N, Cassandrini D, Denora PS, Criscuolo C, Belarbi S, Orlacchio A, Jonveaux P, Silvestri G, Hernandez AM, De Michele G, Tazir M, Mariotti C, Brockmann K, Malandrini A, van der Knapp MS, Neri M, Tonekaboni H, Melone MA, Tessa A, Dotti MT, Tosetti M, Pauri F, Federico A, Casali C, Cruz VT, Loureiro JL, Zara F, Forlani S, Bertini E, Coutinho P, Filla A, Brice A, Santorelli FM. Spastic paraplegia with thin corpus callosum: description of 20 new families, refinement of the SPG11 locus, candidate gene analysis and evidence of genetic heterogeneity. Neurogenetics. 2006;7:149-56.

11. Stevanin G, Santorelli FM, Azzedine H, Coutinho P, Chomilier J, Denora PS, Martin E, Ouvrard-Hernandez AM, Tessa A, Bouslam N, Lossos A, Charles P, Loureiro JL, Elleuch N, Confavreux C, Cruz VT, Ruberg M, Leguern E, Grid D, Tazir M, Fontaine B, Filla A, Bertini E, Durr A, Brice A. Mutations in SPG11, encoding spatacsin, are a major cause of spastic paraplegia with thin corpus callosum. Nat Genet. 2007;39:366-72.

12. Bouchard JP, Richter A, Mathieu J, Brunet D, Hudson TJ, Morgan K, Melançon SB. Autosomal recessive spastic ataxia of CharlevoixSaguenay. Neuromuscul Disord. 1998;8:474-9.

13. Riverol M, Samaranch L, Pascual B, Pastor P, Irigoyen J, Pastor MA, de Castro P, Masdeu JC. Forceps minor region signal abnormality "ears of the lynx": an early MRI finding in spastic paraparesis with thin corpus callosum and mutations in the spatacsin gene (SPG11) on chromosome 15. J Neuroimaging. 2009;19:52-60.
14. Depienne C, Stevanin G, Brice A, Durr A. Hereditary spastic paraplegias: an update. Curr Opin Neurol. 2007;20:674-80.

15. Dreha-Kulaczewski S, Dechent P, Helms G, Frahm J, Gärtner J, Brockmann K. Cerebral metabolic and structural alterations in hereditary spastic paraplegia with thin corpus callosum assessed by MRS and DTI. Neuroradiology. 2006;48:893-8.

16. Aghakhanyan G, Martinuzzi A, Frijia F, Vavla M, Hlavata H, Baratto A, Martino N, Paparella G, Montanaro D. Brain white matter involvement in hereditary spastic paraplegias: analysis with multiple diffusion tensor indices. Ajnr Am J Neuroradiol. 2014;35:1533-8.

17. Engert JC, Berube P, Mercier J, Doré C, Lepage P, Ge B, Bouchard JP, Mathieu J, Melançon SB, Schalling M, Lander ES, Morgan K Hudson TJ, Richter A. ARSACS, a spastic ataxia common in northeastern Quebec, is caused by mutations in a new gene encoding an 11.5-kb ORF. Nat Genet. 2000;24:120-5.

18. Bouchard JP, Barbeau A, Bouchard R, Bouchard RW. Autosomal recessive spastic ataxia of Charlevoix-Saguenay. Can J Neurol Sci. 1978;5:61-9.

19. Gomez CM. ARSACS goes global. Neurology. 2004;62:10-1.

20. Embiruçu EK, Martyn ML, Schlesinger D, Kok F. Autosomal recessive ataxias. 20 types and counting. Arq Neuropsiquiatr. 2009;67:1143-56.

21. Takiyama Y. Sacsinopathies: sacsin-related ataxia. Cerebellum. 2007;6:1-7.

22. Martin MH, Bouchard JP, Sylvain M, St-Onge O, Truchon S. Autosomal recessive spastic ataxia of Charlevoix-Saguenay: a report of MR imaging in 5 patients. AJNR Am J Neuroradiol. 2007;28:1606-8.

23. Van Damme P, Demaerel P, Spileers W, Robberecht W. Autosomal recessive spastic ataxia of Charlevoix-Saguenay. Neurology. 2009;72:1790.

24. Anheim M, Fleury M, Monga B, Laugel V, Chaigne D, Rodier G, Ginglinger E, Boulay C, Courtois S, Drouot N, Fritsch M, Delaunoy JP, Stoppa-Lyonnet D, Tranchant C, Koenig M. Epidemiological, clinical, paraclinical and molecular study of a cohort of 102 patients affected with autosomal recessive progressive cerebellar ataxia from Alsace, eastern France: implications for clinical management. Neurogenetics. 2010;11:1-12.

25. Terracciano A, Foulds NC, Ditchfield A, Bunyan DJ, Crolla JA, Huang S, Santorelli FM, Hammans SR. Pseudodominant inheritance of spastic ataxia of Charlevoix-Saguenay. Neurology. 2010;74:1152-4.

26. Bouchard JP. Reccesive spastic ataxia of Charlevoix-Saguenay. In: de Jong JMBV, editor. Hereditary neuropathies and spinocerebellar atrophies. Handbook of clinical neurology. Amsterdam: Elsevier; 1991. pp. 451-9.

27. Baets J, Deconinck T, Smets K, Goossens D, Van den Bergh P, Dahan K, Schmedding E, Santens P, Rasic VM, Van Damme P, Robberecht W, De Meirleir L, Michielsens B, Del-Favero J, Jordanova A, De Jonghe P. Mutations in SACS cause atypical and late-onset forms of ARSACS. Neurology. 2010;75:1181-8.

28. Synofzik M, Soehn AS, Gburek-Augustat J, Schicks J, Karle KN Schüle R, Haack TB, Schöning M, Biskup S, Rudnik-Schöneborn S, Senderek J, Hoffmann KT, MacLeod P, Schwarz J, Bender B, Krüger S, Kreuz F, Bauer P, Schöls L. Autosomal recessive spastic ataxia of Charlevoix Saguenay (ARSACS): expanding the genetic, clinical and imaging spectrum. Orphanet J Rare Dis. 2013;15:41.

29. Prodi E, Grisoli M, Panzeri M, Minati L, Fattori F, Erbetta A, Uziel G, D’Arrigo S, Tessa A, Ciano C, Santorelli FM, Savoiardo M, Mariotti C. Supratentorial and pontine MRI abnormalities characterize recessive spastic ataxia of Charlevoix-Saguenay. A comprehensive study of an Italian series. Eur J Neurol. 2013;20:138-46.

30. Brodal P. The central nervous system: structure and function. Oxford: Oxford University Press; 2003. 
31. Gazulla J, Benavente I, Vela AC, Marin MA, Pablo LE, Tessa A, Barrena MR, Santorelli FM, Nesti C, Modrego P, Tintoré M, Berciano J. New findings in the ataxia of Charlevoix-Saguenay. J Neurol. 2012;259:869-78.

32. Gazulla J, Vela AC, Marín MA, Pablo L, Santorelli FM, Benavente I, Modrego P, Tintoré M, Berciano J. Is the ataxia of
Charlevoix-Saguenay a developmental disease? Med Hypotheses. 2011;77:347-52.

33. Goller K, Reihle C, Meckel S. Infantile ascending hereditary spastic paralysis (IAHSP): MRI findings in a 15-year-old girl. Rofo. 2013;185:768-9. 